\title{
Ortsregister
}

Alabama $\quad 61,74,77 f ., 82,86,103,125,131$, 134, $137-139,141,148$ f., 151, 156, 164, 183, 195, 385, 419

Alaska $10,75,151,425 \mathrm{f}$.

Amoskeag, NJ 343

Arkansas $61,68,74,90,139,164,171,173$, 183, 192, 197, 385

Atlanta, GA 61, 192, 195

Australien 215, 232

Baltimore $4,30,36,64,110 \mathrm{f} ., 194,233$, 237, 241, 257, 259, 264, 269, 275 f., 292, 302, 327-329, 336, 347, 413

Baton Rouge, LA $\quad 24,60,66,74 f ., 77,89$, $94,104,113,119,125$ f., 137, 192, 321, 417

Berkeley 8, 24, 62, 110, 122, 146, $188 \mathrm{f}$., 202, 223, 229, 231, 235, 249, 326, 401, 426, 431, 442, 447

Berlin 2, 8, 21, 23, 45, 51f., 55f., 70, $80 \mathrm{f}$, $86,96,116,129,140,205,209,215$, 239, 264, 359, 364, 369, 430

Birmingham, AL 191, 195 f., 272, 419

Black Hawk, MS 187

Black Hills 219

Boston, MA 4, 6, 65, 73, 82, 101, 103, 108, $123,138,150,161,174,201,205,213$, 216, 226f., 230, 233, 235, 238, 240, 244, 250f., 271, 273, 285-287, $328 \mathrm{f}$., $344,352,364,368,371,412,424 \mathrm{f}$., 429, 431, 448, 453

British Columbia 151

Brunsbüttel 37

Bud Bagsak 451

Bud Dajo 451

California $8,24,62,118,122,175 \mathrm{f} ., 179 \mathrm{f}$. , 183, 188 f., 191, 202f., 208f., 214, 223, 231, 235, 238, 241, 244, 249, 272, $325 \mathrm{f}$., $338,349,354,361,376,427,430,442$

Canton, $\mathrm{OH} \quad 339,421$

Castle Clinton 231

Chattanooga, TN 138
Cheyenne, WY 171, 218f., 228

Chicago, IL 18, 22, 45, 49, 62f., 107, 113, 115, $126 \mathrm{f} ., 160,162,174,184,186,212$, $225,227-231,233,235-237,239,251$, 253, 258, 262, 265, 284-287, 296, 302, $314 \mathrm{f} ., 321,327,329,331,333,336,347$, 349, 352f., 355, 359-361, 364, 366, 369, 371f., 374, 378, 382, $384 \mathrm{f} ., 388$, 390, 392, 399, 401-405, 408, 417, 419, 422, 443, 448, 452

China $6,18,36,175,207,231,238,320 f$. , 338f., 373, 408, 444, 458

Cleveland, $\mathrm{OH} \quad 245,276$

Cold Spring Habor 240

Colorado 173, 175, 183, 217-219, 304, 316, 345 f., 391, 413, 440

Coney Island, NY 229

Cripple Creek 345f., 391, 405

Dänemark 241

Deadwood, SD 209

Detroit, MI 174, 232, 278, 374

Deutschland 2, 5, 8, 50, 153f., 175, 188, 205, 207, 231, 235f., 289, 296, 347 f., 355, 369, 446, 457

Dominikanische Republik 152, 426

East Baton Rouge, LA $93 \mathrm{f}$.

Ellis Island 231f., 237

England 5f., 36, 72f., 79, 148 f., 156, 191, 198, 207, 231, 239, 247, 259, 262, 329, 343, 359, 384, 447f., 457

Fall River, MA 342

Florida $58,60,71,74,80,89,131,139$, 148, 153, 165 f., 183, 195, 197, 305, 321, 434

Frankreich $\quad$, 149 f., 188, 245, 329, 348, 427, 444, 457

Galizien 238

Genf 148

Ә OpenAccess. (C) 2019 M. Michaela Hampf, publiziert von De Gruyter. (cc) BY-NC-ND Dieses Werk ist lizenziert unter der Creative Commons Attribution-NonCommercial-NoDerivatives 4.0. 
Georgia $21,58,61,71-74,80,84,103$, 106, 128, 136-139, 158, 163f., 195, 200 f., 213 f., 343, 362, 365f., 378, 385, 390, 418, 434

Großbritannien 148f., 151, 156, 205, 235, 263, 427

Guam 441, 444, $448 \mathrm{f}$.

Guantanamo 19

Hawai’i 427

Hell's Kitchen 243

Hester Street 235

Homestead, PA 102, 170, 175f., 179f., 184, 215, 246, 275, 357-359, 392-398, 405, 413

Idaho $175,183,210,220,264,304,391 \mathrm{f}$. Illinois $\quad 60,67,78,99,107 f ., 125,131,147$, 150, 167, 183, 198f., 203, 208, 230, 233, 236 f., 251, 255, 258, 261, 266, 290, 304, $313 \mathrm{f} ., 320 \mathrm{f} ., 325,327,331,333,346$, $349,351,360,363-365,371 \mathrm{f} ., 381-$ $383,385,390-392,399,403,418,422$

Indiana $96,105,145,157,174,179,183$, 198, 241, 246, 257, 305, 397, 400, 412, 422

lowa $181,183,198 f ., 201,203,264,285$, $306,317,347,388,416$

Irland 137, 175, 207, 231, 235, 250, 321, 355

Italien $150,175,207,231,235,238,247$, 321, 337

Japan $16,189,207,231,431,458$

Jolo 452

Kalabrien 238

Kanada 131, 151, 156, 174 f., 220, $231 \mathrm{f} ., 346$

Kansas 158, 165, 173, 177f., 183f., 189, $228,230,269,301,317,329,378,382$, $384,388,390,392,411,414,417,437$, 450

Kentucky 88 f., 113, 143, 150, 163, 179, 194f., 199, 230, 272, 285, 299, 321, 390

Kiatschow Bay 444

Kuba 428f., 435-438, 440-443, 445, 448 f., 451
Little Rock, AK 139

Los Angeles, CA $114,218,228,231,347$, 361

Louisiana $10,24,58,60,66,68,70,74 \mathrm{f}$., 77, $88 \mathrm{f} ., 92-95,97 \mathrm{f} ., 104,112 \mathrm{f} ., 119$, 125 f., 131, 134, 137, $139-141,159,165$ f., 183, 192, 195, 197, 305, 321, 385, 417

Lower Easr Side 241

Lower East Side 235

Luzon 449

Madison County, FL 139

Maine 420, 425, 435, 437, 443, 452

Manchester, NH 358

Marengo County, AL 138

Martinsburg 327

Massachusetts $\quad 68,86,114,152,237,247$, 250, 258, 302, 307, 334 f., 342f., 359, $365,368,377,390,447$

Massillon, $\mathrm{OH} \quad 412$

Memphis, TN 113

Mexiko 149f., 155, 175, 185, 189, 207, 231

Mindanao 450

Minneapolis, MN 5, 56, 214, 230, 237, 246f., 253, 269, 328, 348, 381, 384, $392,396,401$

Mississippi $\quad 5,24,69 f ., 82 \mathrm{f} ., 85,88,94$, 97, 103f., 107, 120, 127, 131-133, 139, $146,158,162-165,183,187,192,201$, 203, 207, 253, 318, 417

Missouri $\quad 87,132,166,172,183,185,193$, 197, 228, 249, 307, 331f., 381, 452

Montana $175,177,183,212,219 \mathrm{f} ., 228$

Nebraska 318

Neuseeland 232

Nevada $183,209,228,316,347$

New York 1-9, 11f., 14-16, 18, 20-23, 32 f., $35-39,42,46,51 f ., 58 f ., 61 f ., 64$, $66,68,70-74,76-82,84-87,89 \mathrm{f}$, 92, 102, $104 \mathrm{f} ., 108-110,114,116 \mathrm{f} ., 120$, $123-128,130-135,139 f ., 143-150,155$, $159,162,165-168,172,174,176$ f., 180, 187f., 190, 192-195, 197-200, 203 f., 206, 208-210, 212-214, 216-219, $221-224,226-233,235-244,247-$ $253,256,258-263,265-278,280-$ 
290, 292f., 295-299, 301f., 304-307, 309, 312-315, 317, 321f., 324, $327-$ 329, 331-333, 335-339, 341, $343 \mathrm{f}$., $347-350,352-357,359-364,366-$ $369,372-379,383-385,389-393$, 395-398, 400 f., 403 f., 407 f., $411-413$, 415, 417-419, 421-423, 431-437, 438, 441-444, 446, 448

\section{Nicaragua 442}

North Carolina $\quad 24,67,74,77 \mathrm{f} ., 82,86-88$, 100, 114, 126, 131, 134, 137-139, 150, 164, 187, 189, 194, 197, 218, 230 f., 274, 277, 284 f., 287, 306 f., 354, 381, 386, 417, 421, 425, 450

North Dakota 175, 183, 201, 203

Ohio $66,107,114,120,122 \mathrm{f} ., 145,165,198$, 226, 241, 245, 249, 255, 263, 276, 292, 305, $308 \mathrm{f} ., 312,327,372,396,412 \mathrm{f}$, 419, 422, 433, 443

Oklahoma 155, 172 f., 175, 184 f., 188, 203, 212 f., 215 f., 219, 222f., 256, 385, 391

Omaha, NE $180,228,381,383,415 \mathrm{f}$.

Oregon 166, 183, 203, 214, 220, 453

Österreich 21

Österreich-Ungarn 231, 321, 323

Pearl River 26, 175

Pennsylvania 7, 72, 114, 121f., 154, 157, 190, 196, 199, 219, 232, 247, 252, 268, 270, 274 f., 277, 289, 306, 327f., $330-$ 332, 341, 349, 354, 365f., 397, 447

Philadelphia, PA $7,60,73,77,84,90,106$, 112, $121 \mathrm{f} ., 154,165,180,208,219,228$, 230, 232, 235f., 244, 253, 261, 273, 277, 281, 285, 299, 330 f., 337, 347, 352, 354, 362, 364 f., 382, 403, 409, 434, 447

Philippinen 29, 35, 189, 428-431, 434, 436, 440 f., 443-446, 448f., 451, 453 f., 458

Pittsburgh, PA 115, 174, 196, 222, 246, 253, 274, 277, 286, 294, 321, 330, 350 f., 357f., 392, 394-397, 430, 437

Pointe Coupée Parish, LA 94, 97

Polozk 174

Promontory Point 268, 339
Puerto Rico 434, 436, 443, 445, 447-449, 458

Reading, PA 327

Russland 10, 174 f., 231f., 235, 268, 321, 425

Saint Helena Island, SC 73

San Francisco, CA 122, 208-210, 212, 218, 231, 233, 244, 249, 269, 272, 282, 327, 329, 338, 349, 375f., 401, 413, 440

Sand Creek, CO $218 \mathrm{f}$.

Savannah, GA 61, 84, 193

Scranton, PA 331, 355

South Carolina $58,60,70-74,77,80,84$, $87-89,103-105,109,126,128,132$, 134, 162, 165 f., 190, 195, 218, 362

South Dakota 175, 223, 451

Spanien 29, 375, 380, 408, 423, 426, 429f., $434-438$, 440 f., $443-445,447-$ 449,451

Sulu $\quad 450-452$

Tennessee $61,68,84,100-102,112-114$, $120,135,138,140,164,195,200,452$

Texas $10,30,74,78,82,84,124,133,152$, 158, 163 f., 173, 175, 187f., 195, 197, 210, 212f., 216, 234, 267, 324, 350, 372, $385 f ., 388 f ., 437$

Titatia 453

Tombstone, AZ 209

Türkei 231

Utah $175,183,199,228,268,304,338 \mathrm{f}$.

Vicksburg, MS 164

Virgin Islands 151, 426

Virginia $61,68,73 \mathrm{f} ., 80,83,90,124,137$, 163f., 166, 209, 327, 346

Virginia City, NV 209

Washington, DC $\quad 4 \mathrm{f} ., 18,20,23 \mathrm{f} ., 60,63$, 72f., 78, 82, 86, 92, 100, 106, 108, 110, 113, 116, 128 f., 131, 137 f., 142, 144, 147-152, 157 f., 162, 164-166, 171, 173, 176, 183, 190, 199 f., 203, 205, 210, 212 f., 215, 218, 221, 224, 230, 241, 
247-249, 260, 275, 284, 292, 301, 307 f., 314, 326, 335, 348-350, 355, $357,363,366,385,396,398,401,403$, 407, 413 f., 423, 425 f., 437, 439, 443 f., 448 f., 451

Washington (State) 175, 205

West Virginia 327, 329
Wisconsin 18, 20, 23, 151, 183, 200 f., 225, 230, 244, 305, 307, 352, 360, 432, 451

Wounded Knee, SD $223 \mathrm{f}$.

Wyoming 175, 183, 210, 220, 228, 304

Yazoo, MS 127, 163 
